\title{
(2) OPEN ACCESS \\ Cardiorespiratory fitness in breast cancer survivors: a randomised controlled trial of home-based smartphone supported high intensity interval training
}

\section{Eisuke Ochi (D) , ${ }^{1,2}$ Katsunori Tsuji (D) , ${ }^{1}$ Tomomi Narisawa (D) , ${ }^{1}$ Yoichi Shimizu (D) , ${ }^{3}$ Aya Kuchiba (D) , ${ }^{4,5}$ Akihiko Suto, ${ }^{6}$ Kenjiro Jimbo, ${ }^{6}$ Shin Takayama, ${ }^{6}$ Taro Ueno (i) , ${ }^{7}$ Naomi Sakurai, ${ }^{8}$ Yutaka Matsuoka (D) ${ }^{1}$}

- Additional supplemental material is published online only. To view, please visit the journal online (http://dx.doi. org/10.1136/bmjspcare-2021 003141).

For numbered affiliations see end of article.

\section{Correspondence to}

Professor Yutaka Matsuoka, Division of Health Care Research, Center for Public Health Sciences, National Cancer Center Japan, Chuo-ku, Tokyo, Japan; matsuoka-psy@umin.ac.jp and Associate Professor Eisuke Ochi, Faculty of Bioscience and Applied Chemistry, Hosei University, Tokyo, Japan; ochi@hosei.ac.jp

EO and KT contributed equally.

Received 21 April 2021 Accepted 4 June 2021

Check for updates

(c) Author(s) (or their employer(s)) 2021. Re-use permitted under CC BY-NC. No commercial re-use. See rights and permissions. Published by BMJ.

To cite: Ochi E, Tsuji K, Narisawa T, et al. BMJ

Supportive \& Palliative

Care Epub ahead of

print: [please include Day

Month Year]. doi:10.1136/

bmjspcare-2021-003141

\section{ABSTRACT}

Background A strategy for maintaining and/ or improving cardiorespiratory fitness (CRF) in the growing population of cancer survivors is of major clinical importance, especially in the COVID-19 era. The effect of unsupervised highintensity interval training (HIIT) on increasing CRF in breast cancer survivors is unknown.

Purpose The purpose of this study was to determine whether the newly developed habit-B programme, which involves home-based smartphone-supported HIIT using body weight exercises, improves CRF in early-stage breast cancer survivors.

Methods This single-centre, 12-week, parallelgroup, single-blind, randomised controlled trial involved 50 women with stage I-lla breast cancer, aged 20-59 years, who had completed initial treatment except for hormone therapy. Participants were randomised to either the exercise or control group. The primary outcome was the 12-week change in peak oxygen uptake $\left(\dot{\mathrm{VO}}_{2 \text { peak }}\right)$. Other outcomes included muscle strength, 6 min walk test, resting heart rate, physical activity, fatigue, safety and quality of life.

Results The change in $\dot{\mathrm{V}} \mathrm{O}_{2 \text { peak }}$ and leg strength increased significantly in the exercise group compared with the control group $(p<0.01)$. Changes in other outcomes were not significantly different between the groups.

Conclusion A home-based HIIT intervention can lead to improve CRF and muscle strength in early-stage breast cancer survivors.

\section{INTRODUCTION}

Exercise may play a role in maintaining and improving common cancer-related

\section{Key messages}

What was already known?

- Cardiorespiratory fitness is inversely associated with cardiovascular and allcause mortality in breast cancer survivors.

- Exercise may prevent cancer and improve a variety of cancer health-related outcomes (eg, fatigue, quality of life, functioning, depression and anxiety).

What are the new findings?

- We confirmed that originally developed 12-week home-based smartphonesupported exercise programme (habit-B programme) improved peak oxygen uptake and muscle strength compared with treatment as usual in sedentary breast cancer survivors in Japan.

What is their significance?

- This clinical trial was the world's first home-based high-intensity interval training programme using Information and Communication Technology (ICT) to increases cardiorespiratory fitness and muscle strength in sedentary breast cancer survivors.

- The habit-B programme was designed based on the theory of Bandura for sedentary breast cancer survivors to develop exercise habits.

- Collaborative work with professionals in the field of clinical oncology, exercise science, mental health, rehabilitation, public health, nursing and cancer survivors constitutes a new model for cancer survivorship care.

health outcomes, including physical fitness, quality of life (QOL) and fatigue. ${ }^{1}$ Exercise guidelines for cancer survivors 
issued by multiple international organisations recommend maintaining high levels of physical activity. ${ }^{1-3}$ Previous studies of physical activity and exercise intervention among breast cancer survivors have mainly focused on weight loss, ${ }^{4}$ impairment of endothelial function and lower bone density in postmenopausal women. The exercise and nutrition to enhance recovery and good health for you (ENERGY) trial, an early and representative study conducted in the USA, demonstrated the effectiveness of a home exercise programme and nutritional advice to promote weight loss among breast cancer survivors. ${ }^{4}$ However, because the proportion of overweight adults in Japan is lower than that in the USA, it is not appropriate to adopt the US-developed programme in Japan. It would appear suitable though to develop intervention around cardiorespiratory fitness (CRF), which is the globally important health indicator. ${ }^{5} 6$ In addition, given that the COVID-19 pandemic has made getting sufficient exercise difficult, ${ }^{7}$ there is an urgent need for a homebased exercise programme that can improve CRF. ${ }^{56}$

With the aim of increasing CRF, recent studies have focused on the use of high-intensity interval training (HIIT). HIIT consists of repeated sets of short bursts of high-intensity exercise followed by a rest interval. Most of previous studies were conducted in supervised experimental settings, which appeared to be more beneficial than usual care in cancer survivors at all stages of treatment and aftercare. ${ }^{89}$ However, to our knowledge, no study has investigated the effects of an unsupervised home-based HIIT programme in breast cancer survivors. $^{9}$

Therefore, the aim of this study was to clarify the effects and feasibility of the newly developed homebased high-intensity interval training and behavioral modification using information and communication technology on cardiorespiratory fitness and exercise habits among sedentary breast cancer survivors (habit-B) program. ${ }^{10}$ We hypothesised that the habit-B programme would improve peak oxygen uptake $\left(\dot{\mathrm{V}} \mathrm{O}_{2 \text { peak }}\right)$ compared with a control group.

\section{METHODS}

The study protocol has been described elsewhere. ${ }^{10}$ Briefly, this was a 12-week, parallel-group, singleblind, randomised controlled trial, in which participants were randomly assigned to intervention either with the habit-B programme or control with a smartwatch (Fitbit Versa; Fitbit, San Francisco, California, USA). The protocol was registered with UMIN-CTR (ID: 000036400). Outcomes were assessed at baseline and postintervention (12 weeks). Recruitment was carried out between 27 May 2019 and 14 August 2020 at the National Cancer Center Hospital. Participants were registered using an electronic data capture (EDC) system. After baseline assessments, participants were randomly assigned to one of the two arms by the minimisation method, a form of dynamic computer-generated randomisation, using two prognostic factors: $\dot{\mathrm{V}} \mathrm{O}_{2 \text { peak }}$ and age. The allocation sequence was concealed from the clinical and research staff. The contents of the smartphone app that the participants used during the trial were assigned automatically to either the habit-B programme or control and data security was protected using blockchain technology. ${ }^{11}$ Objective physical measurements were performed by trained and blinded assessors unaware of the assignment information.

\section{habit-B programme interventions}

The habit-B programme involves home-based exercise for 12 weeks (three times per week) with counselling/ exercise guidance and support provided via personalised email and a newly developed smartphone app (mobile push notifications). ${ }^{10}$ The details of these exercises were divided into three stages according to $\dot{\mathrm{V}} \mathrm{O}_{2 \text { peak }}$ at week 0 and the contents of training were designed to increase physical strength incrementally in accordance with the individual's level of strength. Maximum rated perceived exertion during exercise was monitored to evaluate whether the intensity of the exercise performed was appropriate $(18 \pm 2)$. The control group was given with a smartwatch at the start of the intervention. At the end of the study period, all participants were offered the habit-B programme.

\section{Assessment measures}

The primary outcome measure was CRF ( $\left.\dot{\mathrm{V}}_{2 \text { peak }}\right)$. $\dot{\mathrm{V}} \mathrm{O}_{2 \text { peak }}$ was measured using the incremental multistage load method with a bicycle ergometer (Ergomedic 828E, Monark, Stockholm, Sweden) at 60 revolutions per $\min (\mathrm{rpm})$. The test began at 29.4 watt and increased by 14.7 watt per min until exhaustion. When the participant's pedal rotation speed dropped below $55 \mathrm{rpm}$ three times, the test was deemed concluded. Secondary outcomes included a $6 \mathrm{~min}$ walk test, onerepetition maximum (1RM) for leg press, grip strength, chair stand test, resting heart rate and blood pressure, and body composition analysis (MC-780A-N; Tanita, Tokyo, Japan). Also, the following electronic patientreported outcomes (PROs) were obtained: Global Physical Activity Questionnaire score, Cancer Fatigue Scale $^{12}$ and health-related QOL. ${ }^{13}$ Exercise adherence was monitored by input into the app and by heart rate on the smartwatch. The safety of the intervention was assessed through emails, phone calls and input into the app from participants.

\section{Data collection, management, monitoring and auditing}

PRO data were collected using the electronic PRO system on the smartphone app and the EDC was used to manage and monitor the data.

\section{STATISTICAL ANALYSES}

The sample size was calculated by using changes in $\dot{\mathrm{V}} \mathrm{O}_{2 \text { peak }}$ with the exercise intervention as described. ${ }^{10}$ 
Differences between the habit-B group and the control group were compared by an independent two-sample t-test to evaluate the degree of change in all outcomes from baseline to week 12. One-sided tests were used for $\dot{\mathrm{V}} \mathrm{O}_{2 \text { peak }}(\mathrm{mL} / \mathrm{kg} / \mathrm{min})$ and two-sided tests were used for others. Analyses were performed using IBM SPSS Statistics V.26. Data were collected from 30 November 2020 to 27 May 2019. Data analysis, which followed the intention-to-treat approach, was performed from 28 December 2020 to 6 January 2021.

\section{RESULTS}

Fifty breast cancer survivors were randomised to either the habit-B group $(n=25)$ or the control group $(n=25)$ (online supplemental figure 1). One participant in the habit-B group dropped out and five other participants could not complete outcome assessment because of issues related to the COVID-19 pandemic $(n=4)$ and poor physical condition $(n=1)$. Baseline characteristics did not differ between the two groups (online supplemental table 1).

\section{Primary outcome}

Participants in the habit-B programme group showed significantly larger improvement in $\dot{\mathrm{V}}_{2 \text { peak }}$ compared with those in the control group $(0.9$ (95\% CI 0.1 to 1.7$)$ vs $-0.8(95 \% \mathrm{CI}-1.5$ to -0.1$), \mathrm{p}<0.01)$ (table 1$)$. The difference in the amount of change between the two groups was 1.7 (95\% CI 0.7 to 2.7), and the effect size (ES) was 1.06.

\section{Physical function and examination}

Participants in the habit-B programme group also showed a significant improvement in 1RM for leg press $(p<0.01$; table 1$)$. No significant intervention effect was found for the other physical functions (table 1). Resting heart rate was maintained by the habit-B programme $(\mathrm{p}=0.041$; online supplemental table 2$)$.

Table 1 Changes in cardiorespiratory fitness and muscle strength from baseline to week 12

\begin{tabular}{|c|c|c|c|c|c|}
\hline & \multirow{2}{*}{$\begin{array}{l}\text { habit-B }(\mathrm{n}=21) \\
\text { Mean (SD) }\end{array}$} & \multirow{2}{*}{$\begin{array}{l}\text { Control }(\mathrm{n}=23) \\
\text { Mean }(\mathrm{SD})\end{array}$} & \multicolumn{3}{|c|}{ Between-group difference } \\
\hline & & & Mean $(95 \% \mathrm{Cl})$ & ES & $P$ value \\
\hline \multicolumn{6}{|l|}{ Primary endpoint } \\
\hline \multicolumn{6}{|l|}{$\dot{\mathrm{V}} \mathrm{O}_{2 \text { peak }}(\mathrm{mL} / \mathrm{kg} / \mathrm{min})$} \\
\hline Baseline & $25.0(3.0)$ & $24.9(4.6)$ & & & \\
\hline Week 12 & $25.9(2.8)$ & $24.1(4.0)$ & & & \\
\hline Within-group difference & $0.9(1.7)$ & $-0.8(1.6)$ & $1.7(0.7$ to 2.7$)$ & 1.06 & $<0.01$ \\
\hline \multicolumn{6}{|l|}{ Secondary endpoint } \\
\hline \multicolumn{6}{|l|}{ Cardiorespiratory fitness } \\
\hline \multicolumn{6}{|l|}{$6 \mathrm{MWT}(\mathrm{m})^{*}$} \\
\hline Baseline & $586(43)$ & $603(59)$ & & & \\
\hline Week 12 & $615(42)$ & $631(68)$ & & & \\
\hline Within-group difference & $30(30)$ & $29(40)$ & $1(-21$ to 23$)$ & 0.26 & 0.93 \\
\hline \multicolumn{6}{|l|}{$\dot{\mathrm{V}} \mathrm{O}_{2 \text { peak }}(\mathrm{L} / \mathrm{min})$} \\
\hline Baseline & $1.33(0.18)$ & $1.32(0.29)$ & & & \\
\hline Week 12 & $1.39(0.23)$ & $1.29(0.26)$ & & & \\
\hline Within-group difference & $0.06(0.11)$ & $-0.03(0.09)$ & $0.10(0.03$ to 0.16$)$ & 0.95 & $<0.01$ \\
\hline \multicolumn{6}{|l|}{ Muscle strength } \\
\hline \multicolumn{6}{|l|}{ Grip strength (kgw) } \\
\hline Baseline & $22.4(3.8)$ & $23.3(4.2)$ & & & \\
\hline Week 12 & $23.2(4.2)$ & $23.7(3.4)$ & & & \\
\hline Within-group difference & $0.8(2.0)$ & $0.4(2.1)$ & $0.4(-0.9$ to 1.6$)$ & 0.26 & 0.53 \\
\hline \multicolumn{6}{|l|}{ Leg press $1 \mathrm{RM}(\mathrm{kg})^{*}$} \\
\hline Baseline & $93.6(24.3)$ & $98.7(40.6)$ & & & \\
\hline Week 12 & $106.6(26.7)$ & $98.2(30.9)$ & & & \\
\hline Within-group difference & $13.0(15.9)$ & $-0.5(18.1)$ & $13.5(2.9$ to 24.1$)$ & 0.79 & 0.01 \\
\hline \multicolumn{6}{|l|}{ Chair stand test $(\mathrm{s})^{*}$} \\
\hline Baseline & $14.4(3.2)$ & $13.7(2.5)$ & & & \\
\hline Week 12 & $13.5(2.9)$ & $13.3(2.5)$ & & & \\
\hline Within-group difference & $-0.9(2.7)$ & $-0.4(1.8)$ & $-0.5(-1.9$ to 0.9$)$ & -0.21 & 0.50 \\
\hline
\end{tabular}

*The number of 6MWT, Leg press 1RM and Chair stand test were 20 for habit-B and 23 for control.

ES, Effect Size; 6MWT, 6 min walk test; $1 \mathrm{RM}$, one-repetition maximum; $\dot{V}_{2}{ }_{2 \text { eak' }}$ peak oxygen uptake. 


\section{PRO measures}

Compared with the control group, the habit-B programme group showed some beneficial effects in terms of fatigue ( $\mathrm{ES}=0.50, \mathrm{p}=0.09$ ) but not for physical activities or QOL (online supplemental table 3).

\section{Adherence and adverse event}

The adherence rate was $86 \%$ (range 19\%-100\%). No serious (grade $\geq 3$ ) adverse events occurred.

\section{DISCUSSION}

This is first study to reveal that a home-based 12-week HIIT regimen involving body weight exercises improves $\dot{\mathrm{VO}}_{2 \text { peak }}$ as the primary endpoint and maintains resting heart rate as the secondary endpoint in sedentary breast cancer survivors. Given that previous observational studies have demonstrated an association between increased CRF and reduced cancer-related mortality, ${ }^{14}$ it is reasonable to expect that improving $\dot{\mathrm{V}} \mathrm{O}_{2 \text { peak }}$ may improve health-related outcomes in cancer survivors. The present findings related to CRF are consistent with those of a previous meta-analysis study focusing on supervised HIIT in cancer survivors (10 trials; $2.1 \mathrm{~mL} / \mathrm{kg} / \mathrm{min}) .{ }^{15}$ Although the present programme led to slightly less improvement $(1.7 \mathrm{~mL} /$ $\mathrm{kg} / \mathrm{min}$ ) compared with these earlier supervised and lab-based studies, we believe that our home-based programme is promising for cancer survivors as a feasible intervention.

In addition, we confirmed that the habit-B programme improved leg strength (15.6\%), which was similar to that reported in a previous study involving supervised HIIT (13.3\%). ${ }^{6}$ This result might be an important outcome related to avoiding sarcopaenia in the future. Although the habit-B programme did not affect body composition or physical activity, it led to a meaningful reduction in fatigue, which could be a potential target symptom for exercise intervention.

There are two limitations of our study. Given that our results may not be generalisable to all breast cancer survivors, we need to conduct further large-scale multicentre studies. In addition, due to movement control order implemented to contain the spread of COVID19, we had to stop our face-to-face assessments and consequently postponed the outcome measurements except for PRO. A subanalysis demonstrated similar results $\left(\dot{\mathrm{VO}}_{2 \text { peak }}, 1.5,95 \% \mathrm{CI} 0.5\right.$ to $2.5 ; 1 \mathrm{RM}$, $15.7,95 \% \mathrm{CI} 3.6$ to 27.7 ) even when we excluded the participants who could not visit our lab on their scheduled date, suggesting that our programme may not have been substantially affected by the COVID-19 pandemic.

In summary, our findings support the incorporation of home-based and unsupervised exercise programmes into breast cancer survivorship care plans, especially in the COVID-19 era. The strengths of our programme are its efficiency, feasibility and safety in terms of improving CRF by using a home-based setup of e-health technology and unsupervised body weight exercises.

\section{Author affiliations}

${ }^{1}$ Division of Health Care Research, Center for Public Health Sciences, National Cancer Center Japan, Chuo-ku, Tokyo, Japan

${ }^{2}$ Faculty of Bioscience and Applied Chemistry, Hosei University, Koganei, Tokyo, Japan

${ }^{3}$ Division of Nursing, National Cancer Center Hospital, Chuo-ku, Tokyo, Japan

${ }^{4}$ Biostatistics Division, Center for Research Administration and Support, National Cancer Center Japan, Chuo-ku, Tokyo, Japan

${ }^{5}$ Division of Biostatistical Research, Center for Public Health Sciences, National Cancer Center Japan, Chuo-ku, Tokyo, Japan

${ }^{6}$ Division of Breast Surgery, National Cancer Center Hospital, Chuo-ku, Tokyo, Japan

${ }^{7}$ SUSMED Inc, Chuo-ku, Tokyo, Japan

${ }^{8}$ Cancer Solutions, Chiyoda-ku, Tokyo, Japan

\section{Twitter Yutaka Matsuoka@DottoreYutaka}

Acknowledgements The authors wish to thank Professors Uchitomi (National Cancer Centre Japan), Imoto (Kyorin University) and Iwata (Aichi Cancer Centre) and Dr Shimazu (National Cancer Centre Japan) for their generous support and helpful advice. We are grateful for the contributions of Fitbit for research support. The authors also wish to thank the following current or former staff compensated for their efforts in data management and outcome measurement of this project: Ms Akutsu, Dr. Ishino, Ms Mitsuyama, Mr Hirayama and Ms Shimoji. We thank Dr Kinoshita for intellectual input in protocol development. This study was supported by the National Cancer Centre Research and Development Fund (30A-17).

Contributors EO, AK and YM conceived and designed the study. AS, KJ and ST recruited the subjects. KT and AK conducted the data analysis. EO, KT and YM drafted the main text, figure and tables. All authors reviewed the manuscript and contributed to acquisition, analysis and interpretation of data. All authors have approved the completed version of the manuscript. EO and YM are cocorresponding authors for this manuscript.

Funding This study is supported by a National Cancer Centre Research and Development Fund (30-A-17).

Competing interests $\mathrm{EO}$ has received research support from Nippon Suisan Kaisha. AK has received speaking fees from Chugai Pharmaceutical. TU is a president and shareholder of SUSMED. YM has received speaker fees from Suntory, Pfizer, Mochida, Eli Lilly, Morinaga Milk and Cimic.

Patient consent for publication Not required.

Ethics approval The protocol was approved by the Institutional Review Board of the National Cancer Center Japan on 28 February 2019 (No: 2018-274).

Provenance and peer review Not commissioned; externally peer reviewed.

Open access This is an open access article distributed in accordance with the Creative Commons Attribution Non Commercial (CC BY-NC 4.0) license, which permits others to distribute, remix, adapt, build upon this work noncommercially, and license their derivative works on different terms, provided the original work is properly cited, appropriate credit is given, any changes made indicated, and the use is noncommercial. See: http://creativecommons.org/licenses/by-nc/4. $0 /$.

\section{ORCID iDs}

Eisuke Ochi http://orcid.org/0000-0002-2497-0250

Katsunori Tsuji http://orcid.org/0000-0003-0508-6774

Tomomi Narisawa http://orcid.org/0000-0001-9774-875X

Yoichi Shimizu http://orcid.org/0000-0002-5154-090X

Aya Kuchiba http://orcid.org/0000-0002-6786-3527 
Taro Ueno http://orcid.org/0000-0003-3697-4301

Yutaka Matsuoka http://orcid.org/0000-0002-8690-8129

\section{REFERENCES}

1 Schmitz KH, Campbell AM, Stuiver MM, et al. Exercise is medicine in oncology: engaging clinicians to help patients move through cancer. CA Cancer J Clin 2019;69:468-84.

2 Rock CL, Doyle C, Demark-Wahnefried W, et al. Nutrition and physical activity guidelines for cancer survivors. CA Cancer J Clin 2012;62:242-74.

3 Hori M, Matsuda T, Shibata A, et al. Cancer incidence and incidence rates in Japan in 2009: a study of 32 populationbased cancer registries for the monitoring of cancer incidence in Japan (MCIJ) project. Jpn J Clin Oncol 2015;45:884-91.

4 Rock CL, Flatt SW, Byers TE, et al. Results of the exercise and nutrition to enhance recovery and good health for you (energy) trial: a behavioral weight loss intervention in overweight or obese breast cancer survivors. J Clin Oncol 2015;33:3169-76.

5 Rogers LQ, Courneya KS, Anton PM, et al. Effects of the BEAT cancer physical activity behavior change intervention on physical activity, aerobic fitness, and quality of life in breast cancer survivors: a multicenter randomized controlled trial. Breast Cancer Res Treat 2015;149:109-19.

6 Dolan LB, Campbell K, Gelmon K, et al. Interval versus continuous aerobic exercise training in breast cancer survivors-a pilot RCT. Support Care Cancer 2016;24:119-27.

7 Peçanha T, Goessler KF, Roschel H, et al. Social isolation during the COVID-19 pandemic can increase physical inactivity and the global burden of cardiovascular disease. Am J Physiol Heart Circ Physiol 2020;318:H1441-6.
8 Mugele $\mathrm{H}$, Freitag N, Wilhelmi J, et al. High-intensity interval training in the therapy and aftercare of cancer patients: a systematic review with meta-analysis. J Cancer Surviv 2019;13:205-23.

9 Tsuji K, Matsuoka YJ, Ochi E. High-intensity interval training in breast cancer survivors: a systematic review. BMC Cancer 2021;21:184.

10 Tsuji K, Ochi E, Okubo R, et al. Effect of home-based highintensity interval training and behavioural modification using information and communication technology on cardiorespiratory fitness and exercise habits among sedentary breast cancer survivors: habit-B study protocol for a randomised controlled trial. BMJ Open 2019;9:e030911.

11 Hirano T, Motohashi T, Okumura K, et al. Data validation and verification using Blockchain in a clinical trial for breast cancer: regulatory sandbox. J Med Internet Res 2020;22:e18938.

12 Okuyama T, Akechi T, Kugaya A, et al. Development and validation of the cancer fatigue scale: a brief, threedimensional, self-rating scale for assessment of fatigue in cancer patients. J Pain Symptom Manage 2000;19:5-14.

13 Rabin R, de Charro F. EQ-5D: a measure of health status from the EuroQol group. Ann Med 2001;33:337-43.

14 Jones LW, Courneya KS, Mackey JR, et al. Cardiopulmonary function and age-related decline across the breast cancer survivorship continuum. J Clin Oncol 2012;30:2530-7.

15 Wallen MP, Hennessy D, Brown S, et al. High-intensity interval training improves cardiorespiratory fitness in cancer patients and survivors: a meta-analysis. Eur J Cancer Care 2020;29:e13267. 